\title{
Device Equivalence in Integrated Optics
}

\author{
Johan H. Berends, Gert J. Veldhuis, Paul V. Lambeck, and Theo J. A. Popma
}

\begin{abstract}
The concept of device equivalence is introduced. In equivalent devices, the light propagation can be described by identically evolving modal expansions, resulting in identical power transfer ratios. By first applying this concept to a $z$ invariant structure with a low refractive-index contrast it is shown how a normalized coordinate space can be defined in which equivalent structures have exactly the same geometry. Subsequently it is shown how this normalized coordinate space can be defined for $z$-variant integrated optical devices, again provided that the lateral refractive-index contrast is small. This normalization makes it possible to perform numerical device simulations in normalized coordinate space, the results being applicable to a large set of equivalent devices. Furthermore, starting from a known design, it simplifies redesigning that device for use at another wavelength or using other materials significantly, the resulting device being equivalent to the original one.
\end{abstract}

\section{INTRODUCTION}

$\mathbf{N}$ ORMALIZED parameters for three-layer slab-waveguide structures were first introduced by Kogelnik and Ramaswamy [1]. Li and Lit [2] showed that for a general multilayer slab-waveguide structure it is possible to define an extended set of normalized parameters. In both cases, the normalized parameters were used to normalize the dispersion equation. Slab-waveguide structures with equal normalized widths (and equal asymmetry parameters) appeared to have equal normalized effective indices. Hewak and Lit [3] even used these normalized parameters to derive a simple expression relating the waveguide dispersion to the dispersion of the individual layers.

Multilayer slab-waveguide structures have a refractiveindex profile that can be described by a one-dimensional (1-D) function $n(x)$. In integrated optics however, structures consisting of a number of parallel straight waveguides (which are $z$-invariant) have a 2 -D refractive-index profile $n(x, y)$. This profile can be transformed into an equivalent 1-D refractive-index profile using the Effective Index Method (EIM) [4] or a similar one. The previously mentioned normalized parameters can then be used to normalize the dispersion equation for the resulting 1-D profile.

This 1-D profile usually consists of layers having only one of two possible refractive indices: either the structure refractive index $n_{s}$ or the background refractive index $n_{b}$ (with $n_{b}<n_{s}$ ). The index contrast is usually very small $\left[\left(n_{s}-n_{b}\right) /\left(n_{s}+n_{b}\right) \ll 1\right]$.

Manuscript received April 4, 1995. This work was supported by the Dutch Technology Foundation (STW).

The authors are with MESA Research Institute, University of Twente, NL7500 AE Enschende, The Nertherlands.

IEEE Log Number 9414399.
In this paper it is shown that in that case, a normalized coordinate system can be defined, in which so-called equivalent devices have exactly the same geometry. This is very useful because it makes it possible to find equivalent devices starting from an original design. Furthermore, numerical device simulations can then be performed in normalized coordinate space, the results being applicable to a large set of equivalent devices.

Our approach is as follows: first we start by defining device equivalence for integrated optical devices. Next, this concept is applied to a $z$-invariant structure, i.e., a structure consisting of a number of parallel straight waveguides. It is shown how the normalized coordinate space is defined in order to ensure that equivalent parallel straight-waveguide structures have the same geometry. It is also shown how the conventional set of normalized waveguide parameters can be extended with a parameter that normalizes the length of the structure.

Most integrated optical devices can be modeled using the step approximation of Marcuse [5]. The modeled structure then consists of parallel straight-waveguide segments only. Of course, each of these segments can be normalized separately. Fortunately, however, the scaling factors needed to scale the width and length of each segment turn out to be the same for all segments. As a result, the device as a whole can be normalized using only two very simple scaling factors, without explicitly going through the laborious procedure of step approximating the device.

As an example it will be shown how the scaling factors can be derived and used to obtain an equivalent device, starting from a known design. FD-BPM calculations were performed in order to verify the equivalence of the two devices. The results of these calculations are presented, confirming the equivalence of the two devices.

\section{ASSUMPTIONS AND RESTRICTIONS}

In this paper we shall only pay attention to linear devices where the three-dimensional (3-D) refractive-index distribution $n(x, y, z)$ can be transformed into an equivalent 2D refractive-index profile $n(x, z)$ using the Effective Index Method (EIM) [4] or a similar one. This is usually a good approximation provided that the guided system modes are not near cut-off. For our approach to be valid, the resulting 2-D profile may only consist of regions having a refractive index $n_{s}$ (refractive index of the structure) or $n_{b}$ (background refractive index) where $n_{s}>n_{b}$. Power loss due to absorption or scattering must be negligible. Most integrated optical components, such as couplers, $y$-junctions, tapers, etc., can be described very well by such a model.

The refractive-index contrast must be small $\left(n_{s}-n_{b}\right) /\left(n_{s}+\right.$ $\left.n_{b}\right) \ll 1$; a requirement that is, in practice, almost automat- 
ically satisfied when the EIM is applied. The smaller the contrast, the better the approximation that the power ratio between two guided system modes is equal to the square of their electric-field amplitudes. As a bonus, it also makes the difference between TE and TM modes negligible. Therefore, we will only have to consider TE polarized fields in our analysis.

Finally, we shall assume that at any $z$-position, the optical field can be well approximated by a weigited sum of the field profiles of the guided system modes, where the modal expansion is performed in a direction perpendicular to the $z$-axis. Reflections are neglected. For this to be a good approximation, the waveguides need to be nearly parallel to the $z$-axis (paraxial approximation: angles of up to only a few degrees are allowed). Input fields are applied at $z=0$ while the field at $z=L$ is considered to be the output field ( $L$ denotes the device length).

\section{Optical-Device Equivalence}

Two optical devices are equivalent if "similar input fields" yield "similar intensity distributions" inside the two devices. This can be formulated more formally: two optical devices $A$ and $B$ are called equivalent devices if there exist linear coordinate transformations $T_{A}$ and $T_{B}$ from the usual coordinate system $(x, z)$ to a normalized coordinate system $(\hat{x}, \hat{z})$ such that:

- Any input field $E_{A}(x, 0)$ that can be applied to device $A$ can be transformed into a normalized field profile $\hat{E}_{A}(\hat{x}$, $0)$ using transformation $T_{A}$.

- The resulting intensity profile $I_{A}(x, z)$ inside device $A$ can be transformed into a normalized intensity profile $\hat{I}_{A}(\hat{x}, \hat{z})$ using the same transformation $T_{A}$.

- Any input field $E_{B}(x, 0)$ that can be applied to device $B$ can be transformed into a normalized field profile $\hat{E}_{B}(\hat{x}$, $0)$ using transformation $T_{B}$.

- The resulting intensity profile $I_{B}(x, z)$ inside device $B$ can be transformed into a normalized intensity profile $\hat{I}_{B}(\hat{x}, \hat{z})$ using the same transformation $T_{B}$.

- For any input field $E_{A}(x, 0)$ there is a corresponding field $E_{B}(x, 0)$ that has the same normalized field profile and results in the same normalized intensity distribution, i.e., $\hat{E}_{A}(\hat{x}, 0)=\hat{E}_{B}(\hat{x}, 0) \Rightarrow \hat{I}_{A}(\hat{x}, \hat{z})=\hat{I}_{B}(\hat{x}, \hat{z})$.

It is the purpose of this paper to show how the transformation to the normalized coordinate system can be found for a particular device, and how equivalent devices can be found in a systematical way. This will be done by first discussing very simple devices, namely $z$-invariant structures. These structures consist of a number of parallel straight-channel waveguides. Next, it will be shown how $z$-variant structures can be treated.

\section{Z-INVARIANT STRUCTURES}

When the effective index method is applied to a structure consisting of a number of parallel straight-channel waveguides, the resulting 2-D refractive-index profile $n(x, z)$ is also independent of $z$ and can therefore be considered a multilayer slab-waveguide structure. The refractive index of the individual layers equals either $n_{s}$ or $n_{b}$ (see Section II); the width of layer number $i$ is denoted by $w_{i}$.

$\mathrm{Li}$ and Lit [2] examined the properties of multilayer slabwaveguide structures extensively. It is easy to see from their formulas that when the normalized width $V_{i}$ of layer number $i$ is defined as

$$
V_{i}=k_{0} w_{i} \sqrt{n_{s}^{2}-n_{b}^{2}}
$$

and the normalized effective index $b_{m}$ of system mode number $m$ (which has an effective index denoted by $N_{m}$ ) is defined as

$$
b_{m}=\frac{N_{m}^{2}-n_{b}^{2}}{n_{s}^{2}-n_{b}^{2}}
$$

then the dispersion equation of any multilayer slab-waveguide structure can be written in terms of $V_{i}, b_{m}$, and the mode number $m$ only. This means that the normalized effective index of a system mode is determined uniquely by the set of $V_{i}$ parameters. Therefore, all structures with the same set of $V_{i}$ parameters have the same normalized effective index $b_{m}$ for mode number $m$.

Furthermore, $\mathrm{Li}$ and Lit [2] presented general formulas for the electric-field profile $E(x)$ inside a multilayer slabwaveguide structure. Using the normalized waveguide parameters as defined above, their formulas can be somewhat rewritten, resulting in the following expression for the electricfield profile $E_{m}(x)$ of mode number $m$

$$
E_{m}(x)=\sqrt{P_{m} S_{x}} f\left(m, V_{i}, S_{x} x\right) .
$$

The scaling factor $S_{x}$ is defined as

$$
S_{x}=k_{0} \sqrt{n_{s}^{2}-n_{b}^{2}}
$$

where $k_{0}=2 \pi / \lambda$ is the free-space wave vector. $P_{m}$ equals the amount of power that is carried by mode $m$. $f$ is a complicated function of $S_{x} x$, the mode number $m$ and the normalized widths $V_{i}$ only.

Note that the expression for the electric-field profile shows that waveguide structures with equal normalized waveguide parameters $V_{i}$ also have the same electric-field profile for mode number $m$, except for a scaling factor $S_{x}$ in the $x$-direction and an amplitude scaling factor $\sqrt{S_{x}}$.

Also note that the $V_{i}$ are equal to $S_{x} w_{i}$, which means that the widths are scaled by the same scaling factor as the $x$ coordinate itself. It can be deduced from this that the linear transformation for the $x$-coordinate must be

$$
\hat{x}=S_{x} x
$$

resulting in a the following normalized electric-field profile

$$
\hat{E}_{m}(\hat{x})=E_{m}(\hat{x}) \sqrt{\left(S_{x}\right)}=\sqrt{P_{m}} f\left(m, V_{i}, \hat{x}\right) .
$$

So, multimode slab-waveguide structures with equal sets of $V_{i}$ parameters do not only have the same set of normalized effective indices, but they also have the same set of normalized field profiles. This automatically satisfies the first and third requirements for device equivalence.

Equivalent devices must also have the same normalized intensity profiles resulting from the same normalized input 


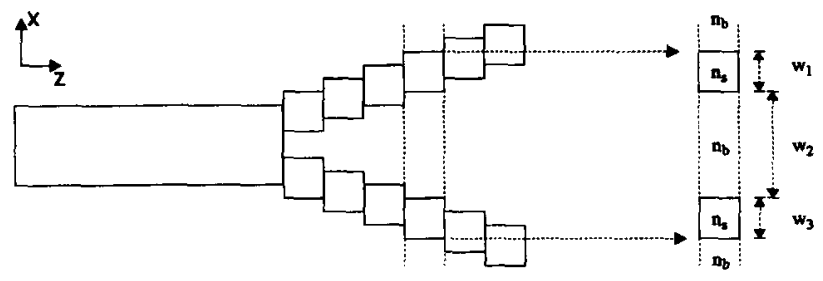

Fig. 1. $Y$-junction modeled by a step approximation.

fields. This must also hold for multilayer slab-waveguides. Since any electric field inside this structure can be written as a weighted sum of the field profiles of the system modes

$$
\begin{aligned}
E(x, z)= & \sum_{m} \sqrt{P_{m} S_{x}} f\left(m, V_{i}, S_{x} x\right) \\
& \cdot \exp \left\{-j k_{0} N_{m} z\right\}
\end{aligned}
$$

the intensity profile is determined by the relative phases of all the system modes. Therefore, the phase difference between two system modes at any normalized $z$-position must be the same in both the usual and normalized coordinate spaces.

In general, the phase difference $\Delta \phi_{m l}$ between any two guided modes $m$ and $l$ of a structure as a function of $z$ can be written as

$$
\Delta \phi_{m l}(z)=k_{0} \Delta N_{m l} z
$$

where $\Delta N_{m l}=N_{m}-N_{l}$ with $m$ and $l$ the mode numbers of the two modes of interest. $N_{m}$ can be expressed as a function of the normalized effective index $b_{m}$ (2)

$$
N_{m}=\sqrt{n_{b}^{2}+b_{m}\left(n_{s}^{2}-n_{b}^{2}\right)} .
$$

Because we have assumed that the refractive-index contrast is small $\left[\left(n_{s}-n_{b}\right) /\left(n_{s}+n_{b}\right) \ll 1\right.$, see Section II] this can be approximated by

$$
N_{m} \approx n_{b}+b_{m}\left(n_{s}-n_{b}\right)
$$

resulting in a phase difference $\Delta \phi_{m l}$ of

$$
\Delta \phi_{m l}(z) \approx k_{0}\left(n_{s}-n_{b}\right) \Delta b_{m l} z
$$

where $\Delta b_{m l}=b_{m}-b_{l}$. Since multilayer slab-waveguide structures with equal sets of $V_{i}$ parameters all have the same $b_{m}$ parameters, they also have the same $\Delta b_{m l}$. Therefore, if the linear transformation in the $z$-direction is defined by

$$
\hat{z}=S_{z} z
$$

with

$$
S_{z}=k_{0}\left(n_{s}-n_{b}\right)
$$

then at equal normalized $z$-positions, the phase differences between all guided system modes are the same in both the usual and normalized coordinate systems. Therefore, their normalized intensity profiles are the same as well.

In order to ensure that two multilayer slab waveguides are equivalent, it is necessary that at any $\hat{z}$-position their normalized intensity profiles are the same in the normalized coordinate space $(\hat{x}, \hat{z})$. This must be particularly true at the outputs of the two waveguides. Now note that, although the length of structure $A$ may be $L_{A}$ and that of structure $B L_{B}$, the normalized length $\hat{L}$ of the two structures must be the same. It is easy to see from (12) what the definition of this normalized length should be

$$
\hat{L}=k_{0}\left(n_{s}-n_{b}\right) L .
$$

Summarizing, two $z$-invariant structures (consisting of a number of parallel straight waveguides) are equivalent if:

- they have the same set of $V_{i}$ parameters

- they have the same normalized lengths $\hat{L}$

provided that they satisfy the assumptions made in Section II. In the next section we shall show how $z$-variant structures can be handled.

\section{Z-VARIANT STRUCTURES}

Most $z$-variant integrated optical devices can be modeled using a step approximation [5], provided that the waveguides remain almost parallel to the $z$-axis. The model then consists of a number of parallel straight-waveguide segments only. Fig. 1 shows an example of a $y$-junction approximated by straightwaveguide segments. Each of the waveguide segments can be transformed to the normalized coordinate system $(\hat{x}, \hat{z})$ using the transformations described in the previous section. This would of course be a very cumbersome procedure, if it were not for the fact that the scaling factors in the $x$ - and $z$-directions are a function of $\lambda, n_{s}$, and $n_{b}$ only. This means that each segment is scaled by the same factor. Therefore, the complete device can easily be transformed as a whole to the normalized coordinate system, using only the simple scaling factors $S_{x}$ and $S_{z}$.

Of course, this transformation to a normalized coordinate system is only meaningful if the local mode expansion at each of the interfaces is not affected by the transformation. This is only true if the values of the overlap integrals between guided modes of two adjacent segments are conserved. The overlap integral $O_{m n}^{P Q}$ between two system modes $m$ and $n$ of two adjacent segments $P$ and $Q$ can be defined as

$$
O_{m n}^{P Q}=\int_{-\infty}^{\infty} E_{m}^{P}(x) E_{n}^{Q}(x) d x .
$$

Similarly, the overlap integral between the same two system modes $m$ and $n$ of the same two segments in normalized coordinate space can be defined as

$$
\hat{O}_{m n}^{P Q}=\int_{-\infty}^{\infty} \hat{E}_{m}^{P}(\hat{x}) \hat{E}_{n}^{Q}(\hat{x}) d \hat{x} .
$$

Using (3) and (6) it follows that the value of the overlap integral is certainly preserved after transformation

$$
\begin{aligned}
O_{m n}^{P Q}= & \int_{-\infty}^{\infty} \sqrt{P_{m}^{P} S_{x}} f^{P}\left(m, V_{i}^{P}, S_{x} x\right) \\
& \cdot \sqrt{P_{m}^{Q} S_{x}} f^{Q}\left(n, V_{i}^{Q}, S_{x} x\right) d x \\
= & \int_{-\infty}^{\infty} \sqrt{P_{m}^{P}} f^{P}\left(m, V_{i}^{P}, \hat{x}\right) \\
& \cdot \sqrt{P_{m}^{Q}} f^{Q}\left(n, V_{i}^{Q}, \hat{x}\right) d \hat{x} \\
= & \hat{O}_{m n}^{P Q} .
\end{aligned}
$$




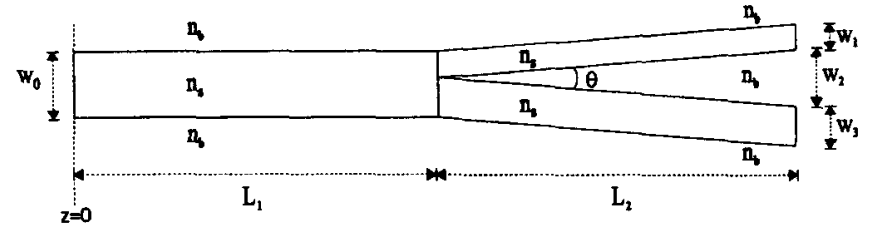

Fig. 2. Geometry of the $y$-junction.

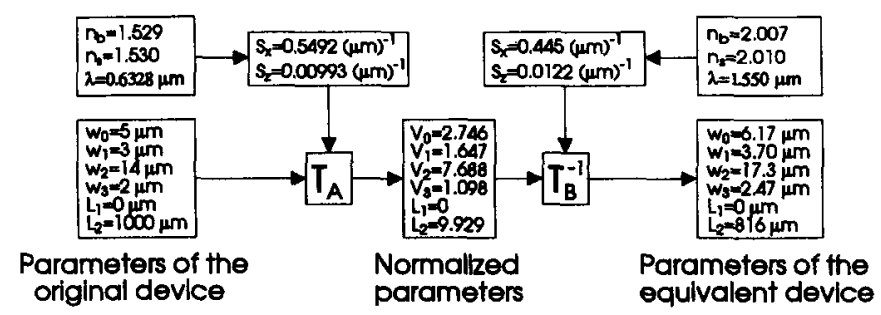

Fig. 3. Calculation scheme for designing an equivalent device.

This result is also consistent with the observations of Baets and Lagasse [6], who noted from numerical simulations that the overlap integral at waveguide discontinuities only depends on the normalized waveguide parameters.

It is now easy to find equivalent devices from the normalized coordinate space. All that has to be done is to choose $\lambda, n_{s}$, and $n_{b}$ for a new device. The scaling factors $S_{x}$ and $S_{z}$ then determine the geometry of the equivalent device.

Although approximating a general device by parallel straight-waveguide segments was necessary to prove device equivalence, equivalent devices can be obtained without going through this procedure. The device must be thought of as consisting of parallel straight-waveguide segments, but the scaling factors can be derived and equivalent devices can be found without doing this explicitly. An example will be given in the next section.

\section{EXAMPLE}

As an example, we shall determine the scaling factors $S_{x}$ and $S_{z}$ for the $y$-junction shown in Fig. 2 and use them to design an equivalent device. The parameters of the original $y$-junction are tabulated in the left-hand side of Fig. 3. This figure also illustrates the process of designing an equivalent device.

The normalized parameters are calculated in the following way. First, using the refractive indices $n_{b}$ and $n_{s}$ of the original device and the wavelength $\lambda$, the scaling factors $S_{x}$ and $S_{z}$ are determined using (4) and (13), respectively. Next, the geometry of the $y$-junction is transformed to normalized coordinate space by multiplying all widths by $S_{x}$ and all lengths by $S_{z}$ in accordance with (5) and (12). Note that this simple procedure, in Fig. 3 denoted by $T_{A}$, is all it takes to transform a device to the normalized coordinate space.

Our next goal is, of course, the design of an equivalent device. First of all, we have to choose the wavelength and the refractive indices of the equivalent device. Equations (4) and (13) can then be used to determine the scaling factors $S_{x}$ and $S_{z}$. The normalized geometry is transformed to the usual coordinate system by dividing all normalized widths by $S_{x}$ and

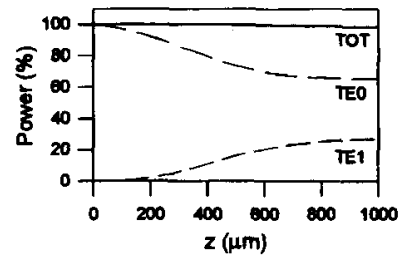

(a)

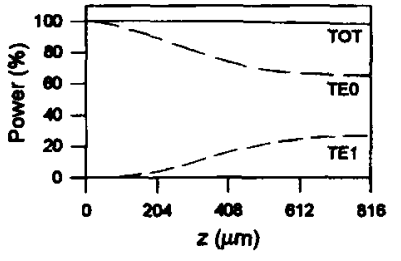

(b)
Fig. 4. Power evolution inside the (a) original and (b) equivalent device.

all lengths by $S_{z}$. This procedure is denoted by $T_{B}^{-1}$ in Fig. 3 . It is an inverse operation because we perform a transformation from normalized coordinates back to the usual ones. The resulting geometry of the equivalent device is tabulated in the right-hand side of Fig. 3.

Simulations with the Finite Difference Beam Propagation Method (FDBPM) [7] using second-order boundary conditions [8] were performed in order to verify the equivalence of the two devices. A TE0 mode was applied to the input of the device at $z=0$. The distribution of power over the two system modes as a function of $z$ is displayed in Fig. 4 for both the original and equivalent devices. The difference between the two results at equal normalized $z$-positions is never more that $0.5 \%$.

\section{SUMmaRY}

In this paper the concept of device equivalence was introduced. In equivalent devices, the light propagation can be described by identically evolving modal expansions, resulting in identical power transfer ratios.

It was shown how a normalized coordinate space can be defined for integrated optical devices, provided that only two refractive indices are involved $\left(n_{b}\right.$ and $\left.n_{s}\right)$ and provided that the refractive-index contrast is small. These requirements are not very restrictive, because application of the effective index method to a three-dimensional structure usually yields a twodimensional structure that satisfies these conditions.

The normalized coordinates were defined in such a way that equivalent devices have the same geometry in the normalized coordinate system. The transformations from the usual coordinate system to the normalized coordinate system appeared to be very simple; only two simple scaling factors [ $S_{x}=k_{0} \sqrt{n_{s}^{2}-n_{b}^{2}}$ and $\left.S_{z}=k_{0}\left(n_{s}-n_{b}\right)\right]$ were required to normalize a device in both the $x$ - and the $z$-direction.

One of the great advantages of working with normalized coordinates is that the results of numerical device simulations performed in this normalized coordinate system are not only applicable to the device under investigation but to a large set of equivalent devices as well. Furthermore, it is now very easy to redesign a device for use at another wavelength or using other materials. This was illustrated by the example in Section VI where a $y$-junction was normalized and an equivalent device was designed. FDBPM simulations confirmed the equivalence of the two devices.

\section{REFERENCES}

[1] H. Kogelnik and V. Ramaswamy, "Scaling rules for thin-film optical waveguides," Appl. Opt., vol. 13, no. 8, pp. 1857-1862, 1974. 
[2] Y. F. Li and J. W. Y. Lit, "General formulas for the guiding properties of a multilayer slab waveguide," Opt. Soc. Am. A, vol. 4, no. 4, pp. $671-677,1987$.

[3] D. W. Hewak and J. W. Y. Lit, "Generalized dispersion properties of thin-film waveguides," Appl. Opt., vol. 25, no. 12, pp. 1977-1981, 1986.

[4] H. Kogelnik, "Theory of dielectric waveguides," in Integrated Optics, T. Tamir, Ed. New York: Springer-Verlag, 1975, ch. 2

[5] D. Marcuse, "Radiation losses of tapered dielectric slab waveguides," Bell Syst. Tech. J., vol. 49, pp. 273-290, 1970.

[6] R. Baets and P. E. Lagasse, "Calculation of radiation loss in integratedoptic tapers and Y-junctions," Appl. Opt., vol. 21, no. 11, pp. 1972-1978, 1982.

[7] Y. Chung and N. Dagli, "An assessment of finite difference beampropagation method," IEEE J. Quantum Electron., vol. 26, pp. 1335-1339, 1990.

[8] H. J. W. M. Hoekstra, G. J. M. Krijnen, and P. V. Lambeck, "New formulation of the beam propagation method based on the slowly varying envelope approximation," Opt. Comm., vol. 97, pp. 301-303, 1993.

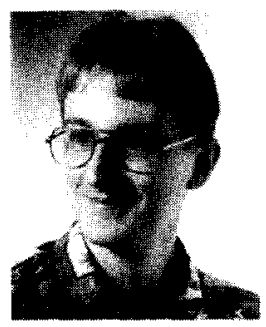

Johan H. Berends was born in Apeldoorn, The Netherlands, on February 7, 1969. He received the M. Sc. degree in electrical engineering from the University of Twente, The Netherlands, in $1992 . \mathrm{He}$ is currently persuing the $\mathrm{Ph}$. D. degree, working on integrated optics narrowband fliters.

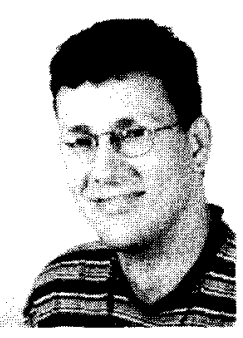

Gert J. Veldhuis was born in Nieuwolda, The Netherlands, in 1970. He received the M. Sc. degree in applied physics from the University of Twente, The Netherlands, in 1994. He is presently working towards the Ph.D. degree in the field of integrated optics for sensor applications.

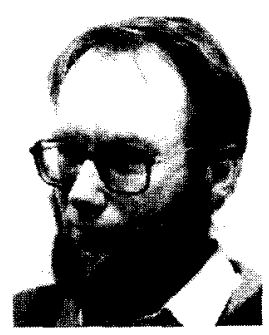

Paul V. Lambeck received the M.Sc. degree in physical chemistry from the University of Amsterdam in 1964. In the same year he joined the University of Twente, starting in the field of ferroelectric materials, a study which resulted in a Ph.D. thesis.

Since 1984 he has heen an Associate Professor in the Lightwave Device Group of the MFSA Research Institute of the University of Twente. His current interest is in the field of integrated optics and concentrates mainly on optical sensors and nonlinear devices.

Theo J. A. Popma was born in Heerenveen, The Netherlands, in 1949. He received the M.Sc. and $\mathrm{Ph} . \mathrm{D}$. degrees from the University of Groningen in 1966 and 1970 , respectively.

During 1970 and 1971 he was at the IBM Thomas J. Watson Research Center in Yorktown Heights, New York. In 1971 he joined the Philips Research Laboratory in Eindhoven, where he was employed until 1984. In 1983 he was appointed Professor of Materials Science in the Departments of Applied Physics and Electrical Engineering of the University of Twente, The Netherlands. 